\title{
The Role of Social Risk Management in Development: $A$ World Bank View - Reply to Comments
}

\author{
Robert Holzmann and Valerie Kozel
}

We appreciate the careful reading and very thoughtful comments. Both sets of comments raise important and interesting issues that we can only imperfectly respond to given time and space. In our response we highlight three issues: (i) SRM as a conceptual framework; (ii) SRM and chronic poverty; and (iii) SRM, development and growth, and touch briefly on several others.

\section{SRM as a conceptual framework}

Social risk management is a conceptual framework about the importance of risk and of risk

management instruments for poverty reduction and development. The proposed conceptualisation emphasises risk (including uncertainty) that can be easily translated into a loss of income (also human capital, and by extension other important outcomes), and stresses the key strategies and arrangements for dealing with risk. SRM undoubtedly has a bias toward economic interpretation and much of our SRM work in the Bank is in this vein. But as a framework, SRM is open to alternative specifications. In our view, much or all of what has been proposed in the comments can be accommodated within the framework. Which of the complementary or alternative specifications (or hypotheses) should be introduced and selected is in the end - as in any science - an empirical question.

As a framework SRM can be compared to the life cycle concept, which states that individuals or households make decisions about e.g. consumption, investment, labour force participation over a longer time horizon. Alternative specifications such as overlapping generation models with two or more generations, infinite time horizon and hence more dynastic views are alternative abstractions that prove useful for different questions. Which of these abstractions is more useful is again an empirical issue, or at least it should be.

Against this background, alternative conceptualisations of SRM are very much welcome and encouraged. And quite definitely other disciplines such as anthropology, sociology and psychology can bring a lot to the discussion. Our familiarity with economics, with its strong analytical underpinnings and, perhaps most importantly, empirical foundations and emphasis on hypothesistesting, are responsible for the bias toward economic interpretations by the authors and the World Bank. Economic science has made important inroads in the understanding of risk and individual decision-making that go beyond expected utility maximisation by borrowing from other sciences, e.g. as demonstrated by behavioural economics and more specifically behavioural finance. For example, the strong empirical results from the latter have already made crucial contributions to the design of pension policy and the structuring of auction processes. The application in other and more informal settings is only starting.

The openness of the SRM approach to empirical validation applies equally to the rights-based approach and the call for minimum provisions. Whether the human rights approach is the best or only feasible approach for guaranteeing basic provisions for the chronic poor and for securing long-term political sustainability of social protection must at the end be left to empirical testing. There are strong arguments in this direction and the Bank has started a new programme of work on empowerment and legislated rights as a crucial 
development instrument. This work needs to be supported by clear-cut analysis and empirical work to avoid ideological under- and over-tones. The reference to Sir Charles Popper and the importance of hypothesis-testing and falsification is more than cultural proximity by one of the authors. It differentiates science from pre-science in the identification of the best development instruments.

\section{SRM and chronic poverty}

A crucial question since the inception of SRM has been its relevance for addressing chronic poverty as compared to transient poverty. Indeed, SRM by itself is quite likely less relevant for dealing with the structural determinants of poverty; this requires policy interventions well beyond social protection. But the SRM framework is conjectured to be still very relevant for addressing chronic poverty - for those who risk falling into chronic poverty or were born therein.

As Lawrence Haddad stated, risks do not necessarily happen one at a time. If repeated and/or bunched, the realm of systemic and idiosyncratic, economic and non-economic risks could overwhelm the (often limited) ability of individuals and households to manage these risks and thus run the danger that they are dragged into a low-level equilibrium trap from which escape may be impossible. One important albeit often overlooked approach for preventing individuals and households from descending into extreme poverty is to think in terms of 'redundancy', i.e. the role of multiple layers of risk management instruments that can come into effect once a prior layer is exhausted. Having multiple layers of risk management plans sounds attractive, but such 'redundancy' or back-up plans come at a price. And thus far we have a limited understanding of how best to structure them beyond the call for social safety nets.

As correctly observed by Guenther et al. income security is not attained by stand-alone savings mechanisms and insurance programmes. In order to reach those at the bottom, much more comprehensive instruments are needed that go beyond traditional social protection instruments. $A$ critical element for any move out of chronic poverty is certainly employment and a good job. For this reason the Social Protection Unit (and the Bank more broadly) has refocused their attention on job creation and employment as a critical transmission mechanism between economic growth and poverty reduction.
Overall, there is no disagreement that dealing with chronic poverty in an effective manner requires interventions from many disciplines and even the best SRM instruments can only provide one dimension to addressing a problem. But the claim stands that SRM contributes to a more sustainable solution and hence goes beyond the statement that the only problem of the chronic poor is the lack of resources.

\section{SRM, development and growth}

SRM has made considerable inroads at the World Bank and beyond. But as with any concept that has natural appeal, it tends to be taken for granted once it is out. And as with many concepts at the interface of economic and social issues, progress is slow and much more needs to be done. We have been pleased to observe IDS' interest in the approach including alternative specifications and views, and we hope there will be more work in this area.

One of the important consequences of introducing the SRM concept was to move the discussion about social protection from a focus on consumption expenditure to one with important investment content that helps build and conserve human capital investment. It challenged the conventionally held view - both inside and outside the Bank - that social protection is for rich countries only. This was assisted by a growing and broadly shared understanding that risk and the absence of appropriate risk management instruments matter for development and progress in poverty reduction. It has strengthened the claim that risk management is crucial for sustainable and equitable economic growth, although empirical evidence still remains patchy.

The SRM approach also gives more scope in the design of public policies to the type of risks and the diversity of strategies and arrangements to address them. Getting the priorities right requires a good empirical understanding of the key risks that individuals are exposed to, coupled with the understanding that the risk hit list in the developing world is typically not led by one of ILO's nine social contingencies. It also helps to expand the typically backward-looking poverty analysis by more forwardlooking vulnerability analysis and efforts to operationalise the concept of the vulnerability. Such Risk and Vulnerability Assessments of different form and sophistication have helped to better identify the drivers of vulnerability-to-poverty. They have also 
helped to focus the minds of policymakers and garner wider political support for social protection.

To conclude: SRM has helped to stimulate a broader global focus on social protection; increasing interest and thinking on the part not only of the Bank but also other international and domestic partners, e.g. ILO, other UN organisations, bilaterals and research institutes (such as IDS), as well as country partners has further helped to shape and focus the discussion. Despite various critiques, we would still claim that the framework has helped to move the discussion from advocacy toward testable hypothesis - a crucial achievement as the development debate moves toward evidence-based policy. 